\title{
SARS-CoV-2 Infection Presenting with Hyperglycemia and Ketosis: A Case Series of Three Diabetic Patients
}

\author{
Choro A Kayina ${ }^{1}$, Souvik Maitra ${ }^{2}$, Rahul K Anand ${ }^{3}$, Bikash R Ray ${ }^{4}$, Dalim K Baidya ${ }^{5}$, Rajeshwari Subramaniam ${ }^{6}$
}

Keywords: Coronavirus disease 2019, Diabetic ketoacidosis, Diabetes mellitus.

Indian Journal of Critical Care Medicine (2020): 10.5005/jp-journals-10071-23595

The novel coronavirus (SARS-CoV-2) infection is known to cause coronavirus disease 2019 (COVID-19) pneumonia and commonly presents with fever, cough, and dyspnea. ${ }^{1}$ However, atypical presentation may not be uncommon and a case of diabetic ketoacidosis (DKA) has been reported. ${ }^{2}$ We are hereby reporting a series of three cases where SARS-CoV-2 infection was associated with hyperglycemia and ketosis without substantial acidemia.

All three patients in our series were female and known diabetic on regular follow-up, aged 35 years (patient 1), 55 years (patient 2 ), and 56 years (patient 3 ). All the three patients presented with fever, cough, and shortness of breath of 2-4 days and severe hypoxemia (oxyhemoglobin saturation $80-85 \%$ ) at the time of admission to emergency screening intensive care unit (ICU). All the three patients had bilateral infiltrates in chest X-ray and bilateral shred sign and pleural thickening in lung ultrasound. Two patients required endotracheal intubation within 6 hours of arrival in ICU (patients 1 and 2) and another patient required intubation after 30 hours of ICU admission for increasing dyspnea and hypoxia. At presentation, significant hyperglycemia was present but arterial $\mathrm{pH}$ was close to normal range in all the three patients (Table 1). All the three patients had hyperlactatemia (lactate $>3 \mathrm{mmol} / \mathrm{L}$ ) and ketone bodies were present in urine. However, baseline serum creatinine values were within normal limit. All the three patients were managed with intravenous crystalloid administration (normal saline and ringers lactate; $3.5,2.5$, and $4 \mathrm{~L}$ in first 24 hours), insulin infusion, and replacement of potassium as indicated. SARS-CoV-2 infection was confirmed by reverse transcription polymerase chain reaction (RT-PCR) of nasopharyngeal swab and endotracheal aspirate and they were treated with methylprednisolone, lowmolecular-weight heparin, and hydroxychloroquine as per institute protocol and insulin infusion for sugar control. However, patients 1 and 2 succumbed to severe acute respiratory distress syndrome (ARDS) and refractory hypoxemia within 72 hours. The other patient had a prolonged hospital stay and was finally discharged.

Angiotensin-converting enzyme 2 (ACE2), a key enzyme in the renin-angiotensin aldosterone system (RAAS), is highly expressed in the pancreas. SARS-CoV-2 may enter into pancreatic islet cells through ACE2 receptors and may directly cause beta-cell injury. ${ }^{3}$ However, it has been found that ACE2 expression may be downregulated in COVID-19 ${ }^{4}$ and this downregulation of ACE2 after viral entry can lead to unopposed angiotensin II impeding further insulin secretion. ${ }^{5}$ These factors may contribute to the precipitation of DKA in diabetic patients with COVID-19.

Fluid resuscitation is an integral part of DKA management. However, aggressive fluid resuscitation can cause further pulmonary edema in COVID-19, compounded by angiotensin II induced increase
${ }^{1-6}$ Department of Anesthesiology, Pain Medicine and Critical Care, All India Institute of Medical Sciences, New Delhi, India

Corresponding Author:Dalim KBaidya, Department of Anesthesiology, Pain Medicine and Critical Care, All India Institute of Medical Sciences, New Delhi, India, Phone: +9111-26593743, e-mail: dalimkumar.ab8@ gmail.com

How to cite this article: Kayina CA, Maitra S, Anand RK, Ray BR, Baidya DK, Subramaniam R. SARS-CoV-2 Infection Presenting with Hyperglycemia and Ketosis: A Case Series of Three Diabetic Patients. Indian J Crit Care Med 2020;24(11):1141-1142.

Source of support: Nil

Conflict of interest: None

in pulmonary vascular permeability. ${ }^{6}$ Liberal fluid strategy targeting resolution of academia and closure of anion gap is indicated in DKA, whereas conservative fluid management is advised in COVID-19 pneumonia. Therefore, fluid resuscitation is a difficult task in such cases and we used repeated point-of-care ultrasound findings and blood gas values targeting hemodynamics, cellular, and biochemical parameters. Although hyperglycemia and ketosis resolved, pulmonary condition worsened and invasive mechanical ventilation had to be initiated by the second day in all the three patients. Although this deterioration can be considered primarily due to progression of disease in view of presenting clinical and radiological features and rapid respiratory deterioration in the first two patients, contribution of fluid resuscitation cannot be ignored, particularly in the third patient.

Table 1: Arterial blood gas analysis of the patients at the time of ICU admission

\begin{tabular}{lccc}
\hline & Patient 1 & Patient 2 & Patient 3 \\
\hline $\mathrm{pH}$ & 7.28 & 7.35 & 7.38 \\
$\mathrm{pO}_{2}$ & 59 & 65 & 70 \\
$\mathrm{pCO}_{2}$ & 40 & 44 & 42 \\
$\mathrm{HCO}_{3}{ }^{-}$ & 18.4 & 23.2 & 24 \\
Glucose & 430 & 340 & 550 \\
Sodium & 137 & 136 & 139 \\
Potassium & 3.7 & 3.5 & 4 \\
Chloride & 107 & 102 & 105 \\
Lactate & 5.5 & 3.1 & 3.3 \\
Anion gap & 15 & 14.3 & 14 \\
\hline
\end{tabular}


To conclude, excessive hyperglycemia and ketosis may manifest in diabetic patients with COVID-19 and the emergency and intensive care physicians should measure blood sugar and ketone levels on presentation in all diabetic patients and be careful about fluid management.

\section{References}

1. Huang C, Wang Y, Li X, Ren L, Zhao J, Hu Y, et al. Clinical features of patients infected with 2019 novel coronavirus in Wuhan, China. Lancet 2020;395(10223):497-506. DOI: 10.1016/S0140-6736(20) 30183-5.

2. Chee YJ, Ng SJH, Yeoh E. Diabetic ketoacidosis precipitated by COVID-19 in a patient with newly diagnosed diabetes mellitus. Diabetes Res Clin Pract 2020;164:108166. DOI: 10.1016/j.diabres.2020.1 08166.
3. Yang JK, Lin SS, Ji XJ, Guo LM. Binding of SARS coronavirus to its receptor damages islets and causes acute diabetes. Acta Diabetol 2010;47(3):193-199. DOI: 10.1007/s00592-009-0109-4.

4. Vaduganathan M, Vardeny $O$, Michel T, McMurray JJV, Pfeffer MA, Solomon SD. Renin-angiotensin-aldosterone system inhibitors in patients with COVID-19. N Engl J Med 2020;382(17):1653-1659. DOI: 10.1056/NEJMsr2005760.

5. Carlsson PO, Berne C, Jansson L. Angiotensin II and the endocrine pancreas: effects on islet blood flow and insulin secretion in rats. Diabetologia 1998;41(2):127-133. DOI: 10.1007/s001250050880.

6. Guo J, Huang Z, Lin L, Lv J. Coronavirus disease 2019 (COVID-19) and cardiovascular disease: a viewpoint on the potential influence of angiotensin-converting enzyme inhibitors/angiotensin receptor blockers on onset and severity of severe acute respiratory syndrome coronavirus 2 infection. J Am Heart Assoc 2020;9(7):e016219. DOI: 10.1161/JAHA.120.016219. 